\title{
Inhibition of return in static but not necessarily in dynamic search
}

\author{
Zhiguo Wang AND Kan ZHANG \\ Institute of Psychology, Chinese Academy of Sciences, Beijing, China \\ AND \\ RAYMOND M. KLEIN \\ Dalhousie University, Halifax, Nova Scotia, Canada
}

\begin{abstract}
If and when search involves the serial inspection of items by covert or overt attention, its efficiency would be enhanced by a mechanism that would discourage reinspections of items or regions of the display that had already been examined. Klein (1988, 2000; Klein \& Dukewich, 2006) proposed that inhibition of return (IOR) might be such a mechanism. The present experiments explored this proposal by combining a dynamic search task (Horowitz \& Wolfe, 1998, 2003) with a probe-detection task. IOR was observed when search was most efficient (static and slower dynamic search). IOR was not observed when search performance was less efficient (fast dynamic search). These findings are consistent with the "foraging facilitator" proposal of IOR and are unpredicted by theories of search that assume parallel accumulation of information across the array (plus noise) as a general explanation for the effect of set size upon search performance.
\end{abstract}

Inhibition of return (IOR) was first discovered by Posner and Cohen (1984; for a review, see Klein, 2000). Most scholars have explored IOR using the model task pioneered by Posner in which targets requiring simple responses are preceded by nonpredictive cues. Typically, at short cuetarget onset asynchrony (CTOA), response times (RTs) to targets appearing at a previously cued location are shorter than for targets appearing at an uncued location. However, at long CTOA, the pattern is reversed and RTs to targets presented at a cued location are longer than those to targets presented at an uncued location. In their seminal study, Posner and Cohen proposed that the function of IOR is to encourage orienting toward novel items.

Visual search is a widely used paradigm in attention research. In a typical search task, participants are asked to search for a target among a group of distractors. The target may be present or absent in the array of items, and the participant makes a present/absent decision. When the search task is easy, the target "pops out" of the array, and RT is relatively unaffected by the number of items presented (set size). In contrast, when the search task is more difficult, RT increases, usually linearly, with the size of the array, and the slope of the RT $\times$ set size function on target-absent trials is steeper than that on target-present trials. To avoid a theoretical commitment - and although recognizing that using two labels implies a dichotomy when a continuum of difficulty might provide a more accurate characterization - we will refer to these two types of search tasks as "easy" and "difficult," respectively. As for the prototypical pattern of results with difficult search tasks, two broad classes of theory have been advanced to explain the effect of set size on RT. One class of theory (e.g., Broadbent, 1987; Ratcliff, 1978; Townsend, 1974) asserts that information is processed or accumulated in "parallel" across all the items in the array, and a response is issued once enough evidence has been accumulated for either the "present" or "absent" decision. A second class of theory (Treisman \& Gelade, 1980; Wolfe, 1994; Wolfe, Cave, \& Franzel, 1989) asserts that individual items in the array or regions containing a small number of items are inspected serially by an attentional operator to determine whether the item is a target, or whether the region contains one. The linear relationship between RT and set size, and the finding that the slopes for target-absent trials are often approximately twice those of target-present trials, are straightforward consequences of this serial, selfterminating search strategy. (We will refer to these two classes of theory on how difficult search is accomplished as parallel and serial, respectively.) Although serial theories implicitly assume that, once rejected as distractors in difficult search tasks, items are not (or not likely to be) reinspected, originally no explicit mechanism was described that would prevent or discourage such reinspections (but see Koch \& Ullman, 1985).

Klein (1988, p. 430) asked, "How does the serial search mechanism keep track of where attention has been, so that it does not return there again?" Building on Posner and Cohen's (1984) suggestion that IOR decreases the likeli- 
hood that attention would return to previously attended items, Klein proposed that "inhibition of return might help perform this function, perhaps in conjunction with consciously directed search strategies" (p. 430). He tested this proposal with a probe-following-search paradigm (Klein, 1988). On half the trials, shortly after the search task response, a luminance probe was placed either at a location previously occupied by a search display item (on-probe) or at a previously empty location (off-probe). If some IOR-like mechanism facilitated difficult search, after a search response inhibitory tags would be left at some or all locations occupied by distractors, and RTs for on-probes would be longer than RTs for off-probes - a difference we will refer to as on-probe cost. Moreover, this on-probe cost should be smaller, if not absent, following easy (popout) search because, by hypothesis, in pop-out search, the target is detected "preattentively"- that is, without the need for the individual distractors to be inspected. In two experiments, these predictions were confirmed (Klein, 1988). Although some researchers (Wolfe \& Pokorny, 1990) failed to replicate Klein's (1988) findings, Müller and von Mühlenen (2000) and Takeda and Yagi (2000) found clear evidence for inhibitory tags after difficult searches so long as the display items remained visible on the screen at the time the probe was presented, suggesting that the inhibitory tags laid down during difficult search are encoded into object-based, or scene-based, representation of the search array (see also Klein \& MacInnes, 1999; Ogawa, Takeda, \& Yagi, 2002). These findings provide a foundation for Klein's (1988) proposal that the IOR might serve as a foraging facilitator in difficult search (for a review, see Wang \& Klein, in press).

In the present article, we follow the strategy used by Takeda and colleagues (e.g., Ogawa et al., 2002) to explore the boundary conditions for the observance of IOR in search. The dynamic search paradigm developed by Horowitz and Wolfe (1998) is one that, by design, precludes the contribution of any possible memory mechanism (including IOR) that might discourage reinspections in visual search. In a typical dynamic search task, items in the display continuously change their positions at relatively short intervals. This manipulation would make useless any memorial mechanism that keeps track of the movements of attention to assist serial search, including IOR, because the target at one point in time can be in the location where a distractor had been previously. In this study, we will use - for the first time, as far as we knowthe probe-following-search paradigm developed by Klein (1988) to determine whether IOR is generated in dynamic search.

There are at least three possible strategies that a participant might use in dynamic search: (1) the serial search strategy described above; (2) the sit-and-wait strategy, keeping attention fixed on a small region of the display in the expectation that, eventually, the target (if present) will appear there (von Mühlenen, Müller, \& Müller, 2003); or (3) the parallel accumulation strategy described above in which, essentially, the attentional beam is broadened to encompass the entire array. In both the sit-and-wait and parallel strategies, attention is essentially stationary until a target that may capture attention is detected. In contrast, in the serial strategy, an attentional operator moves from item to item until the target is found or the observer decides it was not present. Therefore, given the generally accepted notion that IOR is an aftermath of orienting (for a review, see Klein, 2000), IOR should be observed following dynamic search if participants are using a serial search strategy in this paradigm. On the other hand, if IOR is not observed following dynamic search, this would suggest that participants are using either the parallel or the sit-and-wait strategies.

In Experiment 1, we sought to replicate the finding of IOR (Klein, 1988; Müller \& von Mühlenen, 2000; Takeda \& Yagi, 2000) using a static probe-following-search task. In Experiment 2, we combined a fast dynamic search task (items exchanged locations every $120 \mathrm{msec}$ ) and a probe task to determine whether the IOR effect would or would not be found. In Experiment 3, a dynamic search task with a slower rate of change (items exchanged locations every $420 \mathrm{msec}$ ) was combined with the probe procedure. Because the probe logic developed by Klein (1988) depends on a clear distinction between "on" and "off" probes, we chose to use a dynamic search task in which the locations where items are displayed from one "frame" to the next remain the same, whereas the target (when present) and distractors randomly exchange places (see, e.g., Kristjánsson, 2000).

\section{EXPERIMENT 1}

Using the probe-following-search paradigm developed by Klein (1988), Müller and von Mühlenen (2000) and Takeda and Yagi (2000) found IOR in visual search when search items remained visible during probe presentation. The purpose of Experiment 1 was to replicate these findings with new materials to set the stage for Experiments 2 and 3 , which utilized the same materials while employing a dynamic search task.

\section{Method}

Participants. In all experiments reported in this article, participants reported normal or corrected-to-normal visual acuity. ${ }^{1}$ Each participant was paid 20 yuan $/ \mathrm{h}$ for his or her participation. In Experiment 1 , the participants were 20 college students ( 9 male).

Apparatus and Materials. The experiment was conducted in a dimly lit laboratory. Stimuli were presented on a 17 -in. CRT monitor with resolution set to $800 \times 600$ pixels and refresh rate set to $85 \mathrm{~Hz}$. The participants viewed the monitor from a distance of about $65 \mathrm{~cm}$.

The search display consisted of 6 or 12 stimuli (set size). Distractors were the letter " $\mathrm{T}$ " $\left(0.7^{\circ} \times 0.7^{\circ}\right)$ randomly rotated $0^{\circ}, 90^{\circ}$, $180^{\circ}$, or $270^{\circ}$. In the difficult search task, the target was the letter "L" $\left(0.6^{\circ} \times 0.7^{\circ}\right)$ rotated $90^{\circ}$ clockwise. In the easy search task, the target was a circle $\left(0.7^{\circ} \times 0.7^{\circ}\right)$. The probe was an empty black square $\left(1.0^{\circ} \times 1.0^{\circ}\right)$. Items in the search display occupied randomly chosen locations defined by three invisible circles with 4,8 , or 12 locations, respectively. The diameters of the three invisible circles were $5^{\circ}, 7.8^{\circ}$, and $10.6^{\circ}$.

Procedure. The procedure is illustrated in Figure 1. At the start of each trial, a fixation cross was presented at the center of the monitor. After 1,000 msec, while the fixation cross remained visible, search display was presented either until the participants issued a response or until 7,000 msec had passed. Participants were asked to make 


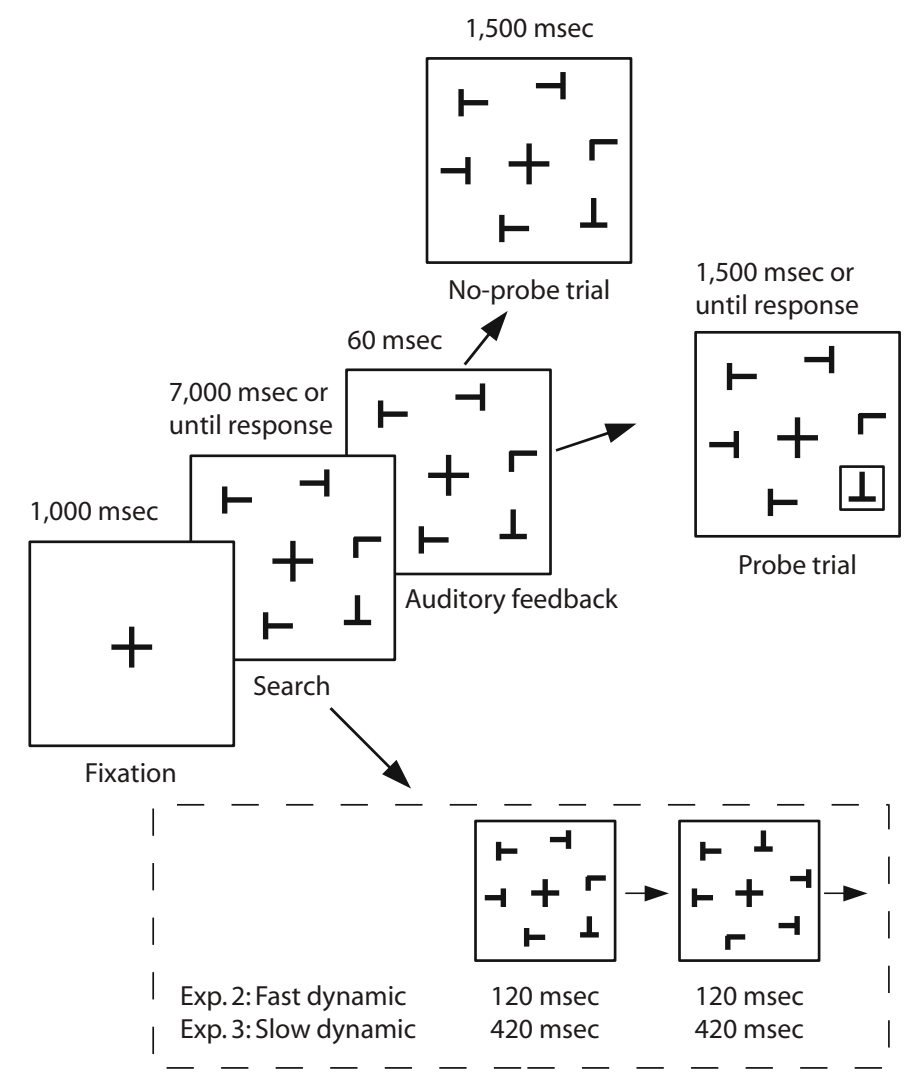

Figure 1. Procedure of Experiments 1, 2, and 3. Figures in the dashed box show that items in the search display randomly exchange locations every 120 msec (Experiment 2) or 420 msec (Experiment 3). The probe illustrated in this figure is an on-probe.

target-present and target-absent responses by pressing, respectively, " 1 " (index finger) and " 2 " (middle finger) on the numeric keypad with the right hand. The response was followed by an auditory feedback signal (a high-pitched beep denoted a wrong reaction; a lowpitched beep denoted a correct reaction), which lasted for $60 \mathrm{msec}$. The search display continued after the auditory feedback. Upon termination of the feedback ( $60 \mathrm{msec}$ after the search response), a probe (empty black square) was added to the search display on half of the trials. The postsearch display (with or without the probe) was terminated after $1,500 \mathrm{msec}$, or when the participant responded by pressing "F" with the left index finger.

Both the difficult and easy search tasks consisted of 192 trials: target (present or absent) $\times$ set size $(6$ or 12) $\times 48$ trials. Each task was divided into two blocks of 96 trials each. The four blocks were organized into two sequences (ABBA and BAAB) and were balanced across participants. On half of the trials in each target $\times$ set size condition, the search task response triggered the presentation of an empty black square (probe). Fifty percent of such stimuli were presented at locations occupied by the search display distractors (onprobes), and 50\% were presented at empty locations (off-probes); probes never appeared at locations previously occupied by a target. ${ }^{2}$ Prior to each block, participants were exposed to a 32-trial practice. Thus, each participant completed a total of 512 trials in a session lasting approximately $35 \mathrm{~min}$.

Probe-detection performance analysis. Although our focus will be on probe RT, miss and false alarm rates are also reported. Because on-probes are added to locations occupied by the search items but off-probes are placed in empty locations, this physical difference may cause a higher miss rate for on-probes. As with RT, if IOR operates in difficult search, we would expect this on-off dif- ference of miss rate to be larger for difficult than for easy search. An increase in false alarms might reflect a speed-accuracy tradeoff, but because false alarms occur in the absence of a probe, it is not possible to associate false alarms with on- versus off-probe performance.

Methods for probe RT analysis were the same as those used by Müller and von Mühlenen (2000). If IOR operates in difficult visual search, RTs for on-probes should be greater than those for off-probes (on-probe cost), and this on-probe cost should be greater in difficult search than in easy search (differential on-probe cost), where IOR is not expected to be present. Differential on-probe cost, which would be signaled by a significant interaction between task and probe condition, was taken as a measure of IOR effect. As in Müller and von Mühlenen, probe RTs were not analyzed if preceded by an incorrect search response. Furthermore, under the assumptions of serial selfterminating search, each item in a search array should be visited/ inspected by attention on target-absent trials, whereas, on average, only half of the items would be visited prior to finding the target on target-present trials. If IOR is generated and becomes measurable when attention leaves an item or location, then on target-absent trials, all the array items should be tagged with IOR, and on target-present trials, only half of the items in the array should be tagged with IOR. For this reason, when using the probe-following-search method to explore IOR following search, Klein (1988; Klein \& Taylor, 1994) has emphasized the IOR scores computed from target-absent trials, with the expectation that the IOR score on target-absent trials might be roughly twice that on target-present trials, an expectation that has been confirmed by Wang and Klein (in press) in a meta-analysis of studies that have employed the probe-following-search method developed by Klein (1988). 
Exp. 1: Static

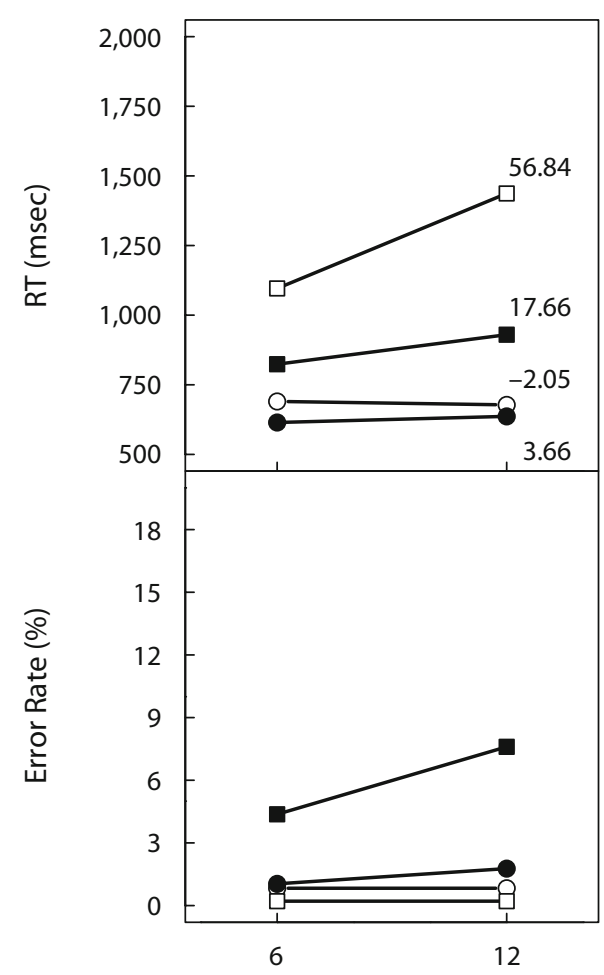

Set Size
Exp. 2: Fast Dynamic

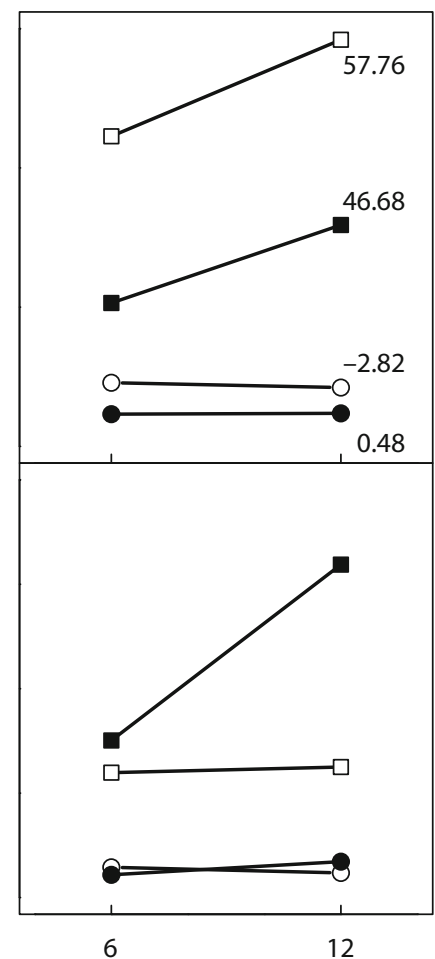

Set Size
Exp. 3: Slow Dynamic

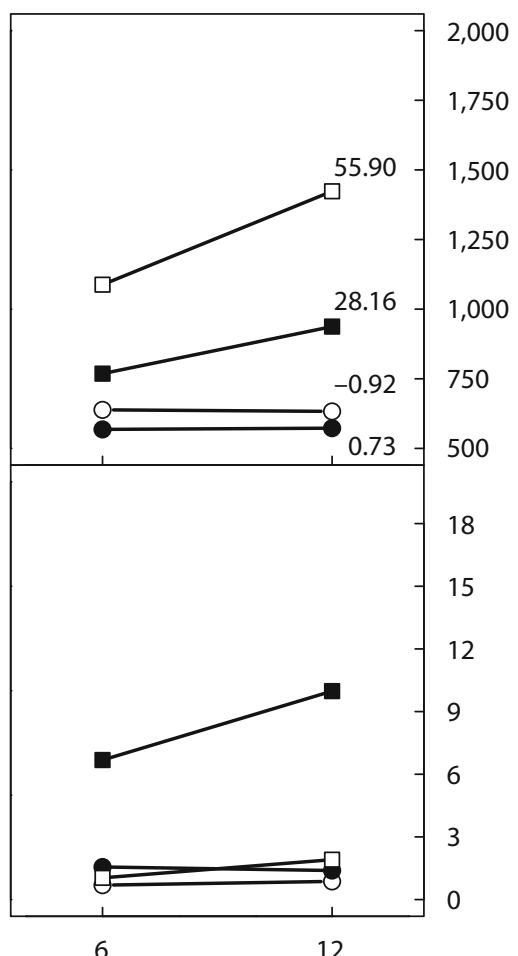

Set Size

Figure 2. Mean response times (RTs) and error rates of Experiments 1, 2, and 3. Circles denote easy search, and squares denote difficult search: Open symbols denote target-absent trials, and filled symbols denote target-present trials. Numbers in the upper-row figures are search slopes (msec/item).

\section{Results}

Search performance. Search performance data (RTs and corresponding error rates) of Experiment 1 are presented in Figure 2.

An ANOVA of the error rates with the variables task (difficult or easy), target (absent or present), and set size (6 or 12) revealed significant main effects of task $[F(1,19)=22.39, p<.001]$, target $[F(1,19)=33.21, p<$ $.001]$, and set size $[F(1,19)=7.38, p<.05]$. Significant interactions were found between task and target $[F(1,19)=$ $20.92, p<.001]$, task and set size $[F(1,19)=4.37, p=$ $.05]$, target and set size $[F(1,19)=4.55, p<.05]$, and task, target, and set size $[F(1,19)=5.20, p<.05]$.

An ANOVA of the search RT data with the variables task, target, and set size revealed significant main effects of task $[F(1,19)=102.29, p<.001]$, target $[F(1,19)=48.55, p<$ $.001]$, and set size $[F(1,19)=84.90, p<.001]$. Significant interactions occurred between task and target $[F(1,19)=$ $39.23, p<.001]$, task and set size $[F(1,19)=86.73, p<$ $.001]$, target and set size $[F(1,19)=26.17, p<.001]$, and task, target, and set size $[F(1,19)=23.97, p<.001]$.

Probe-detection performance. The mean RT, miss rate, and false alarm rate of each condition are presented in the Appendix.

Misses for the probe task following the easy and difficult search tasks were $1.67 \%$ and $2.13 \%$, respectively; the false alarms for these two tasks were $0.84 \%$ and $1.15 \%$, respectively. An ANOVA of miss rates with the variables task (difficult or easy), target (absent or present), set size (6 or 12), and probe (on or off) revealed a significant main effect of set size $[F(1,19)=4.67, p<.05]$ and significant interactions between set size and probe $[F(1,19)=4.69$, $p<.05]$ and between task and probe $[F(1,19)=12.17$, $p<.01]$. This latter interaction may reflect an IOR effect, with $1.77 \%$ greater on-probe costs for misses in the difficult than in the easy search task. There was also a marginally significant interaction between task, target, and set size $[F(1,19)=3.98, p=.06]$; all the other main effects and interactions were not significant (all $F \mathrm{~s}<2.95$, all $p \mathrm{~s}>.10)$. A three-way (task $\times$ target $\times$ set size) ANOVA of the false alarms of the probe task revealed a significant main effect of target $[F(1,19)=5.72, p<.05]$ and a marginally significant interaction between target and set size $[F(1,19)=4.11, p=.057]$; all the other main effects and interactions were not significant (all $F_{\mathrm{S}}<2.27$, all $p \mathrm{~s}>.14)$.

An ANOVA of the probe RTs of Experiment 1 with the variables task, target, set size, and probe revealed significant main effects of task $[F(1,19)=7.70, p<.05]$, target $[F(1,19)=67.60, p<.001]$, and probe $[F(1,19)=$ $28.74, p<.001]$. Significant two-way interactions were observed between task and target $[F(1,19)=5.80, p<$ 
$.05]$ and task and probe $[F(1,19)=11.60, p<.01]$. The latter interaction reflects the finding that the on-probe cost was larger for difficult search $(22.6 \mathrm{msec}$, averaged across target-absent and target-present trials) than for easy search $(7.5 \mathrm{msec})$, suggesting the existence of an IOR effect. Although the three-way interaction between task, target, and probe was not significant $[F(1,19)=$ $0.53, p=.48]$, the trend was for a greater IOR score on target-absent trials $(20 \mathrm{msec})$ than on target-present trials (10 msec; see Figure 3).

\section{Discussion}

Similar to classical difficult and easy search tasks, and as can be seen in Figure 2, the difficult search task used in the present study produced relatively steep search slopes (56.84 and $17.66 \mathrm{msec} /$ item on target-absent and target-present trials, respectively); the easy search task produced very flat search slopes $(-2.05$ and $3.66 \mathrm{msec} /$ item, respectively).

Reflecting IOR in probe-detection performance, the results of Experiment 1 showed significant differential on-probe costs in both RTs and misses. The RT effect was about $15 \mathrm{msec}$ (see Figure 3). Although nonsignificant, a trend for greater IOR on target-absent $(20 \mathrm{msec})$ than on target-present $(10 \mathrm{msec})$ trials was observed.

In summary, the results of Experiment 1 are similar to the pattern of effects reported by Klein (1988), Müller and von Mühlenen (2000), and Takeda and Yagi (2000), and they lay the groundwork for the next two experiments to look for evidence of IOR following dynamic search.

\section{EXPERIMENT 2}

In Experiment 2, we explored whether IOR would be observed following a dynamic search task when items exchanged locations rapidly (every $120 \mathrm{msec}$ ).

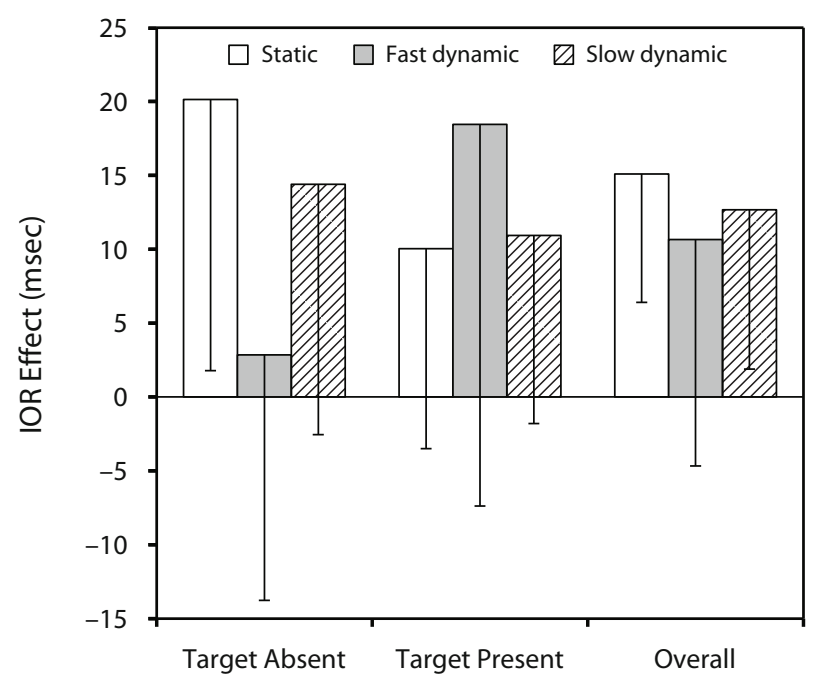

Figure 3. IOR scores of the static (Experiment 1), fast dynamic (Experiment 2), and slow dynamic (Experiment 3) conditions. Error bars denote $95 \%$ confidential intervals.
Method

Participants. Twenty-three college students ( 6 male) participated in Experiment 2.

Stimuli and Procedure. The stimuli used in Experiment 2 were the same as those used in Experiment 1. The procedure used in Experiment 2 was also the same as that in Experiment 1, except that items on the display randomly exchanged places every $120 \mathrm{msec}$ when participants were searching for a target (see Figure 1). However, the first frame of the search display was the same as in Experiment 1 on each trial. Once the participant made the search response (present/absent), the display dynamic was terminated, and the display became static.

\section{Results}

Search performance. Search performance data (RTs and corresponding error rates) of Experiment 2 are presented in Figure 2.

An ANOVA of the error rates of Experiment 2 with the variables task, target, and set size revealed significant main effects of task $[F(1,22)=34.20, p<.001]$, target $[F(1,22)=17.91, p<.001]$, and set size $[F(1,22)=28.88$, $p<.001]$. Significant two-way interactions occurred between task and target $[F(1,22)=15.17, p<.001]$, task and set size $[F(1,22)=30.90, p<.001]$, and target and set size $[F(1,22)=20.82, p<.001]$; a significant threeway interaction was observed between task, target, and set size $[F(1,22)=9.69, p<.01]$.

An ANOVA of the search RTs of Experiment 2 with the variables task, target, and set size revealed significant main effects of task $[F(1,22)=85.71, p<.001]$, target $[F(1,22)=31.20, p<.001]$, and set size $[F(1,22)=26.55$, $p<.001]$. Significant two-way interactions were found between task and target $[F(1,22)=45.20, p<.001]$ and task and set size $[F(1,22)=36.30, p<.001]$; a marginally significant three-way interaction was observed between task, target, and set size $[F(1,22)=3.69, p=.068]$.

Probe-detection performance. The mean RT, miss rate, and false alarm rate of each condition are presented in the Appendix.

Misses following the easy and difficult search task were $0.95 \%$ and $2.26 \%$, respectively, and false alarms were $2.13 \%$ and $1.49 \%$, respectively. An ANOVA of miss rates with the variables task, target, set size, and probe revealed that no main effects or interactions were significant (all $F_{\mathrm{s}}<2.91$, all $p \mathrm{~s}>.10$ ). A three-way (task $\times$ target $\times$ set size) ANOVA of the false alarms revealed a significant main effect of target $[F(1,22)=17.90, p<.001]$ and set size $[F(1,22)=4.75, p<.05]$ and a significant interaction between target and set size $[F(1,22)=7.02, p<.05]$; no other main effects or interactions were significant (all $F_{\mathrm{S}}<1.06$, all $p \mathrm{~s}>.31$ ).

An ANOVA of the probe RTs of Experiment 2 with the variables task, target, set size, and probe revealed significant main effects of task $[F(1,22)=5.62, p<.05]$, target $[F(1,22)=9.43, p<.01]$, and probe $[F(1,22)=$ 4.46, $p<.05]$ and a marginally significant main effect of set size $[F(1,22)=4.14, p<.054]$. Significant twoway interactions were observed between target and set size $[F(1,22)=9.56, p<.01]$. Note that, the two-way interaction between task and probe was not significant 
$[F(1,22)=1.86, p=.19]$, suggesting that no IOR effect was observed in Experiment 2.

\section{Discussion}

As expected, the easy search task produced very flat search slopes $(-2.88$ and $0.48 \mathrm{msec} /$ item for target-absent and target-present trials, respectively). For the difficult search task, although target-absent trials produced a search slope $(57.76 \mathrm{msec} /$ item $)$ similar to that in Experiment 1, responses were slower in Experiment 2, and target-present trials produced a much steeper search slope $(46.68 \mathrm{msec} /$ item) than we observed in Experiment $1(17.66 \mathrm{msec} /$ item). Furthermore, search error rates were higher than in Experiment 1. These findings suggest that the difficult search task with the fast dynamic display used in Experiment 2 was not as efficient as that with static displays in Experiment 1 (see also Klein \& Dukewich, 2006).

Although the task $\times$ probe interaction was not significant, the differential on-probe cost for the hard search task was about $10 \mathrm{msec}$ and was not significantly different from the (significant) 15 -msec effect observed in Experiment 1 $[t(41)=0.47, p=.64]$. Although we believe that it is the absence of IOR in the fast dynamic condition that is the important finding, we recognize that some scholars might not agree. Helping to convince us, and we hope others, is the atypical pattern of IOR scores for target-absent and target-present trials in Experiment 2: Target-absent trials produced a near-zero IOR score (under $4 \mathrm{msec}$ ), whereas target-present trials produced an unexpectedly large (but nonsignificant) IOR score (18 msec; see Figure 3$).{ }^{3}$ As described earlier, if a serial self-terminating search strategy had been used by participants in this experiment, we would have expected to observe a larger IOR score on targetabsent trials than on target-present trials. This abnormal data pattern, together with the nonsignificance of the overall and target-present IOR scores, suggests that IOR is not being generated in the fast dynamic condition.

Why was no clear IOR effect observed in Experiment 2? One class of explanation for this finding might be that the fast-changing search display in Experiment 2 discouraged serial inspection of the individual item-containing locations. Two strategies that might replace serial search in the fast dynamic search task are the "sit-and-wait" strategy (von Mühlenen et al., 2003) and the parallel accumulation strategy (as described earlier). If IOR during search is generated when attention is disengaged from a distractor, then under either of these strategies, IOR would not be generated. Another class of explanation assumes that, despite the inability of memory to be helpful in facilitating search in the fast dynamic search task, observers may still be moving their attention around among the changing items. In this case, the absence of IOR in this condition might be explained by assuming that inhibitory tags are generated in the fast dynamic search task, but the transient change of the display disrupts the tags or precludes their measurement by probes. Although we cannot rule out the latter possibility, we believe that it is unlikely. Müller and von Mühlenen (2000) found significant IOR following search when they used partial offsets (as long as the set size was greater than four), and their partial offsets produced a global transient quite similar, phenomenologically, to the identity change used here.

\section{EXPERIMENT 3}

In Experiment 2, we explored whether IOR would be observed following a fast dynamic search task when items exchanged locations rapidly (every $120 \mathrm{msec}$ ). In Experiment 3 , we explored whether IOR would be observed following a dynamic search task when items exchanged locations more slowly (every $420 \mathrm{msec}$ ).

\section{Method}

Participants. Twenty-four college students (10 male) participated in Experiment 3.

Stimuli and Procedure. The stimuli and procedure of Experiment 3 were the same as in Experiment 2, except that the display frame change rates were slower: every $420 \mathrm{msec}$ (see Figure 1).

\section{Results}

Search performance. Search performance data (RTs and corresponding error rates) of Experiment 3 are presented in Figure 2.

An ANOVA of the error rates of Experiment 3 with the variables task, target, and set size revealed significant main effects of task $[F(1,23)=14.85, p<.001]$, target $[F(1,23)=17.67, p<.001]$, and set size $[F(1,23)=7.56$, $p<.05]$. Significant two-way interactions occurred between task and target $[F(1,23)=11.45, p<.01]$ and task and set size $[F(1,23)=8.00, p<.01]$; a significant threeway interaction was observed between task, target, and set size $[F(1,23)=5.79, p<.05]$.

An ANOVA of the search RTs of Experiment 3 with the variables task, target, and set size revealed significant main effects of task $[F(1,23)=98.93, p<.001]$, target $[F(1,23)=33.80, p<.001]$, and set size $[F(1,23)=$ $36.79, p<.001]$. Significant two-way interactions were found between task and target $[F(1,23)=34.07, p<$ $.001]$, task and set size $[F(1,23)=34.44, p<.001]$, and target and set size $[F(1,23)=14.08, p<.01]$; significant three-way interactions occurred between task, target, and set size $[F(1,23)=21.17, p<.001]$.

Probe-detection performance. The mean RT, miss rate, and false alarm rate of each condition are presented in the Appendix.

Miss rates following the easy and difficult search tasks were $0.95 \%$ and $0.87 \%$, respectively; false alarms were $1.04 \%$ and $1.26 \%$, respectively. An ANOVA of the miss rates of Experiment 3 with the variables task, target, set size, and probe revealed marginally significant main effects of target $[F(1,23)=4.11, p=.054]$ and probe $[F(1,23)=3.64, p=.069]$, a marginally significant interaction between task and target $[F(1,23)=3.45, p=$ .076], and a significant interaction between target and probe $[F(1,23)=9.55, p<.01]$; no other main effects or interactions were significant (all $F \mathrm{~s}<2.63$, all $p \mathrm{~s}>.11$ ). A three-way (task $\times$ target $\times$ set size) ANOVA of the false alarms revealed that all main effects and interactions were not significant (all $F \mathrm{~s}<2.08$, all $p \mathrm{~s}>.16$ ). 
An ANOVA of the probe RTs of Experiment 3 with the variables task, target, set size, and probe revealed significant main effects of target $[F(1,23)=19.24, p<.001]$, probe $[F(1,23)=28.99, p<.001]$, and set size $[F(1,23)=$ $5.73, p<.05]$. A significant two-way interaction was observed between task and probe $[F(1,23)=5.30, p<.05]$, and significant three-way interactions occurred between task, probe, and set size $[F(1,23)=5.69, p<.05]$. The two-way interaction between task and probe suggests that IOR was observed in Experiment 3.

\section{Discussion}

As in Experiment 1, the difficult search task used in Experiment 3 produced steep search slopes (55.90 and $28.16 \mathrm{msec} /$ item on target-absent and target-present trials, respectively), and the easy search task produced very flat search slopes ( -0.9 and $0.7 \mathrm{msec} /$ item, respectively).

For probe-detection performance, significant IOR effect was observed in Experiment 3 (15 and $11 \mathrm{msec}$ for target-absent and target-present trials, respectively; see Figure 3). This was somewhat surprising, because an IOR effect was not observed in Experiment 2, in which a dynamic search task was used, too. It occurred to us, however, that the rate of exchange of items was much slower in this condition-perhaps slow enough for the target to be found in most cases before the first exchange of identities. Close analysis revealed that, given a search rate of around $50 \mathrm{msec} /$ item, $420 \mathrm{msec}$ is more than enough time to inspect 6 items and is almost enough time to inspect 12 items. Thus, unlike in Experiment 2, for which the frame rate was $120 \mathrm{msec}$, it is possible that participants adopted a serial search strategy in Experiment 3, with most of the searching taking place during the first or second frame. The similar pattern of search performance to that obtained with the static display of Experiment 1 supports this interpretation (see Figure 2). Once this possibility is acknowledged, the finding of IOR in this experiment is no longer surprising.

\section{GENERAL DISCUSSION}

It has been over 10 years since Horowitz and Wolfe (1998) developed the dynamic search paradigm as a method for disabling the contribution of memorial mechanisms (such as IOR) to search performance, and over 20 years since Klein (1988) developed the probefollowing-search paradigm for measuring, after a search episode, the lingering inhibitory tags hypothesized to serve as search facilitators by discouraging reinspections. The present study explored whether IOR effect could be found in dynamic search task. Following the on-probe and off-probe logic of Klein (1988), in Experiment 1 we combined a static search task with a probe-detection task, and in Experiments 2 and 3 we combined two dynamic search tasks (Horowitz \& Wolfe, 2003; Kristjánsson, 2000) with a probe-detection task. A significant IOR effect was observed in Experiments 1 (static search) and 3 (slow dynamic), but not in Experiment 2 (fast dynamic).

\section{Search Efficiency of the Dynamic Search Tasks}

The most important aspect of the fast dynamic search task was that any memorial mechanism that keeps track of previous checked items would be relatively useless. To the extent that these mechanisms are important for facilitating search, the efficiency of search should deteriorate in the dynamic condition, unless the search was completed before the identities were exchanged. To test this expectation, we compared the search performance of Experiments 1,2 , and 3 .

Many researchers rely on the slope of the search RT $\times$ set size function as a measure of search efficiency while ignoring or placing little emphasis on the accuracy of performance. And some researchers using a target-present/ target-absent decision have based their assessments of performance efficiency solely on the trials where targets were present. We believe that these reliances are seriously flawed. When an observer is making a two-choice decision (such as whether a target is present or absent in a display) and the experimenter is interested in understanding the efficiency of this decision, it is essential to consider both the speed and the accuracy (Pachella, 1974; Wickelgren, 1977) of all the decisions (Klein \& Dukewich, 2006; Shore \& Klein, 2000). For example, the level of evidence required for making a "present" response will simultaneously control the speed of responding on targetpresent trials, as well as the rate of erroneous (false alarm) responses on target-absent trials. Consequently, focusing on target-present trials alone, a speed-accuracy trade-off produced by a shift in the criterion for making a present response cannot be detected. Plots of mean RT (averaged across target-absent and target-present trials) of the difficult search task, as a function of experiment (Experiment 1,2, or 3) and set size, are shown in Figure 4A, and the corresponding accuracy data are shown in Figure 4B. In order to visualize whether performance across Experiments $1-3$ is equivalently efficient, Figure $4 \mathrm{C}$ plots accuracy on the $y$-axis and RT on the $x$-axis. In this figure, differences along the positive diagonal are characterized by a trade-off between speed and accuracy, whereas differences along the negative diagonal represent unambiguous efficiency differences. As can be seen in Figure 4C, search is much more efficient in the static and slow dynamic tasks than in the fast dynamic task.

Instead of simply plotting RT and accuracy on the same plot, a different approach for quantifying search efficiency is to combine RT and accuracy into a single measure. We chose information transmission rate (Fitts \& Posner, 1967) to serve this purpose. Information transmission rate is calculated by dividing the average amount of information transmitted $\left(H_{\mathrm{T}}\right)$ by the average time it takes to respond (larger numbers represent more efficient performance). Mean information transmission rates are presented in Table 1. From the data presented here, it is clear that search efficiency of the fast dynamic task was inferior to search efficiency of the static search and slow dynamic tasks; the slow dynamic task did not differ much from the static search task. ${ }^{4}$ 


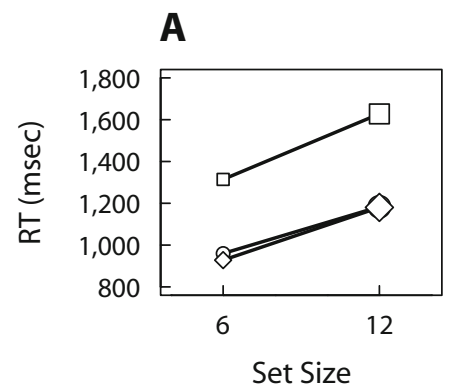

B
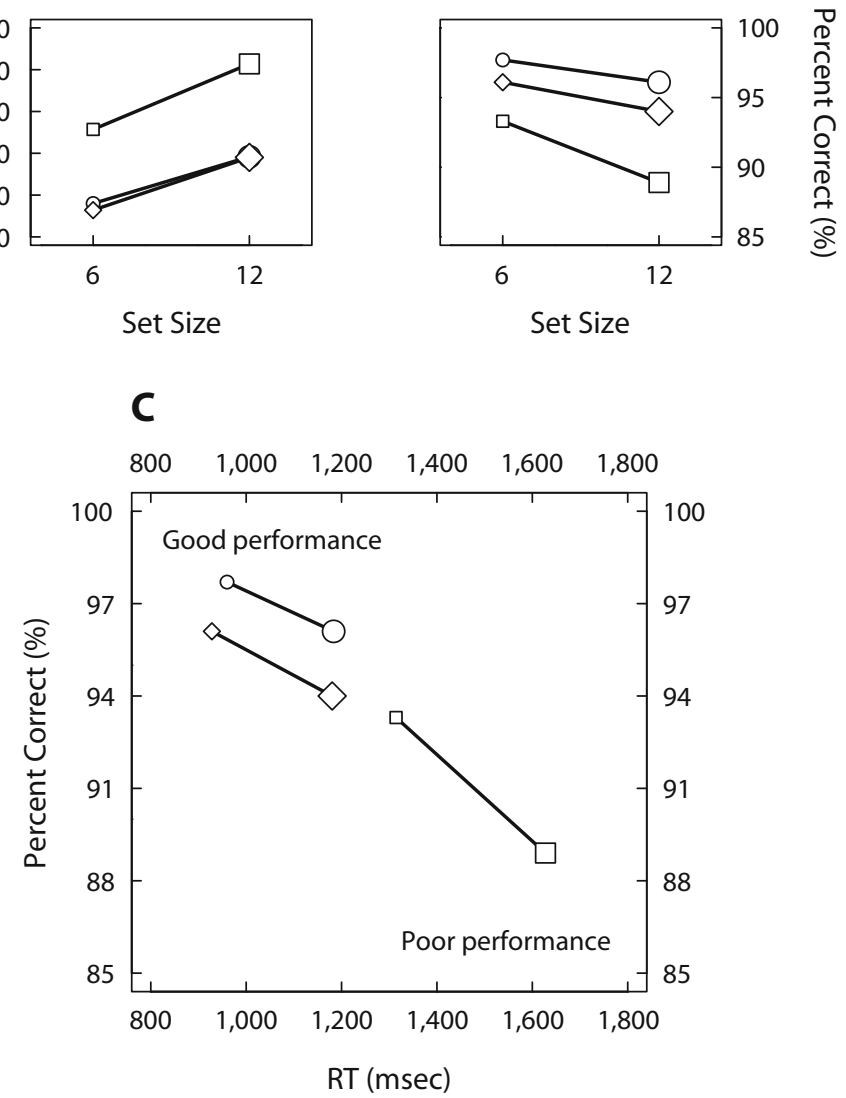

Figure 4. Search performance of the difficult search tasks in Experiments 1, 2, and 3. (A) Response time (RT) (averaged across target-absent and targetpresent trials) for each set size for Experiments 1, 2, and 3. (B) Accuracy (averaged across target-absent and target-present trials) for each set size for Experiments 1, 2, and 3. (C) Mean accuracy (percent correct), plotted against mean RTs for each set size for Experiments 1, 2, and 3. Circles denote Experiment 1 (static search), squares denote Experiment 2 (fast dynamic), and diamonds denote Experiment 3 (slow dynamic). Small symbols denote set size 6, and big symbols denote set size 12 .

\section{Dynamic Search and the "Amnesic" Debate}

In the present study, search efficiency was much greater in the static condition than in the fast dynamic condition. If it is generally the case that fast dynamic displays have a significant negative impact on search efficiency (cf. Klein \& Dukewich, 2006), then the original argument made by Horowitz and Wolfe (1998) - that search in the static condition is amnestic - is undermined, because that argument depended on no loss of search efficiency when intratrial memory was useless. This does not mean, however, that there is no such thing as "amnestic search": quite the contrary. When memory is useless, search is, by definition, amnestic. As described earlier, two search strategies have been described for finding a target in dynamically changing displays, like those used in Experiment 2, that do not depend on an intratrial record of the behavior of attention, because, in both of these strategies, attention need not be moving around. Future studies will be required to determine which of these strategies (sit-and-wait or parallel accumulation) might typically

Table 1

Summary of the Present Study

\begin{tabular}{lccccc}
\hline & & \multicolumn{3}{c}{$\begin{array}{c}\text { Search Efficiency } \\
{\left[H_{\mathrm{T}} / \text { RT (bit/sec) }\right]}\end{array}$} \\
\cline { 3 - 5 } \multicolumn{1}{c}{ Search Task } & Usefulness of Memory & Set Size 6 & Set Size 12 & Overall & IOR \\
\hline Static (Exp. 1) & yes & 0.94 & 0.72 & 0.83 & yes \\
Fast dynamic (Exp. 2) & no & 0.57 & 0.38 & 0.48 & no \\
Slow dynamic (Exp. 3) & $?$ & 0.94 & 0.68 & 0.81 & yes \\
\hline
\end{tabular}


be used in the fast dynamic condition and how efficient these strategies might be.

\section{IOR Effect in the Dynamic Search Tasks}

The logic of dynamic search and key results of our experiments are summarized in Table 1. The "Usefulness of Memory" column is based on the idea that, in static search, intratrial memory (cf. Shore \& Klein, 2000) of what was learned from inspecting each item or region of the display could be used to guide subsequent inspections and that, in the fast dynamic condition, such a memory would be useless. The "?" for our slow dynamic condition indicates that, a priori, we were unsure how disruptive this would be to intratrial memory. Search efficiencies (as measured by information transmission rate) for the static and the slow dynamic conditions were almost the same, and both were much more efficient than those for the fast dynamic condition. Finally, significant IOR effects were observed in the static and slow dynamic conditions, but not in the fast dynamic condition. In our view, the most parsimonious explanation for this pattern of results is that, in the static and slow dynamic conditions, IOR operates as a "foraging facilitator," helping to discourage attention from revisiting inspected locations, whereas, in the fast dynamic condition, IOR is not (or is rarely) generated, because participants are usually using one of two possible strategies in which attention is not moving from item to item.

\section{IOR As Foraging Facilitator in Search}

According to Klein (1988; Klein \& Ivanoff, 2008; Klein \& MacInnes, 1999), the role of IOR in search is not absolute: It does not prevent reinspections. Rather, IOR discourages reinspections by decreasing the likelihood that a previously inspected item in the visual scene will be reinspected. Whether IOR accomplishes this function by operating directly on the saliency map that guides orienting in search (Wolfe, 1994; Wolfe et al., 1989) or at a more motoric level of processing - or at both levels of processing - remains a question for future research. The present study provides supportive evidence (directly and indirectly) for this foraging-facilitator proposal of IOR. First, Experiment 1 replicated Klein (1988) and other studies (Müller \& von Mühlenen, 2000; Takeda \& Yagi, 2000) by finding significant IOR following static search. Second, significant IOR was also observed in the slow dynamic condition, in which search was as efficient as in the static condition. Finally, when the rate of dynamic change was high enough to significantly reduce search efficiency, IOR was not observed.

The foraging-facilitator proposal has been challenged and supported by two recent studies. Hulleman (2009) recently demonstrated that search efficiency was more or less unaffected when the 6 to 18 items in an array moved continuously in random directions. Whereas we agree with Hulleman's conclusion that "items do not have to remain in the same location for visual search to work," (p. 118; see also Shore \& Klein, 2000), we do not agree with the inference that "inhibitory tagging occurs only when the items remain static; whenever the items are mov- ing, other mechanisms support visual search" (p. 119). In arriving at this inference, the idea that IOR in a cue-target paradigm might be coded in object rather than location coordinates was acknowledged by Hulleman, but the capacity of this system to tag multiple moving objects was underestimated. Indeed, combining the probe-followingsearch methodology with randomly moving display items, Ogawa et al. (2002) found evidence of inhibitory tagging in precisely the situation where Hulleman was asserting it to be absent, if not impossible.

Using a method based on Klein and MacInnes's (1999) work, Dodd, Van der Stigchel, and Hollingworth (2009) measured saccadic reaction to probes delivered during inspection of a scene. Supporting the foraging-facilitator proposal, when participants were searching the scene for a target, they found evidence for a relatively long-lasting tendency IOR. Note that this inhibitory effect was dependent on the presence of a search task; simply looking at the scene, even if to memorize it, did not generate the inhibitory tags. This pattern of results suggests that the inhibitory tagging system proposed by Klein (1988) is not an automatic consequence of inspecting a scene. Apparently, oculomotor IOR during scene inspection can be turned on and off, depending on task demands, and it appears to be turned on when participants shift their attention while searching for something in the scene.

\section{AUTHOR NOTE}

We are grateful to the reviewers (Adrian von Mühlenen, Yuji Takeda, and Todd Horowitz) for their constructive advice on an earlier version of this article. The collaboration that is represented here was made possible by a scholarship and travel grant from the China Scholarship Council to Z.W. and a Natural Sciences and Engineering Research Council of Canada Discovery Grant to R.M.K. Correspondence concerning this article should be addressed to Z. Wang, Institute of Psychology, Chinese Academy of Sciences, Beijing, China 100101 (e-mail: wangzg@psych .ac.cn).

\section{REFERENCES}

Broadbent, D. (1987). Simple models for experimentable situations. In P. E. Morris (Ed.), Modeling cognition (pp. 169-185). New York: Wiley.

Dodd, M. D., Van der Stigchel, S., \& Hollingworth, A. (2009). Novelty is not always the best policy: Inhibition of return and facilitation of return as a function of visual task. Psychological Science, 20, 333-339. doi:10.1111/j.1467-9280.2009.02294.x

FitTs, E. M., \& Posner, M. I. (1967). Human performance. Monterey, CA: Brooks/Cole.

Horowitz, T. S., \& Wolfe, J. M. (1998). Visual search has no memory. Nature, 394, 575-577. doi:10.1038/29068

Horowitz, T. S., \& Wolfe, J. M. (2003). Memory for rejected distractors in visual search? Visual Cognition, 10, 257-298. doi:10.1080/ 13506280143000005

Hulleman, J. (2009). No need for inhibitory tagging of locations in visual search. Psychonomic Bulletin \& Review, 16, 116-120. doi:10.3758/PBR.16.1.116

KLEIN, R. [M.] (1988). Inhibitory tagging system facilitates visual search. Nature, 334, 430-431. doi:10.1038/334430a0

KLEIN, R. M. (2000). Inhibition of return. Trends in Cognitive Sciences, 4, 138-147. doi:10.1016/S1364-6613(00)01452-2

KLEIN, R. M., \& DuKEWICH, K. (2006). Does the inspector have a memory? Visual Cognition, 14, 648-667. doi:10.1080/13506280500194022

KLEIN, R. M., \& IVANOFF, J. (2008). Inhibition of return. Scholarpedia, 3, 3650 .

KLEIN, R. M., \& MacInnes, J. (1999). Inhibition of return is a forag- 
ing facilitator in visual search. Psychological Science, 10, 347-352. doi:10.1111/1467-9280.00166

KLEIN, R. M., \& TAYLOR, T. L. (1994). Categories of cognitive inhibition with reference to attention. In D. Dagenbach \& T. H. Carr (Eds.), Inhibitory processes in attention, memory, and language (pp. 113-150). New York: Academic Press.

Koch, C., \& Ullman, S. (1985). Shifts in selective visual attention: Towards the underlying neural circuitry. Human Neurobiology, 4, 219-227.

KRISTJÁNSSON, A. (2000). In search of remembrance: Evidence for memory in visual search. Psychological Science, 11, 328-332. doi:10.1111/ $1467-9280.00265$

MÜller, H. J., \& VON MüHLENEN, A. (2000). Probing distractor inhibition in visual search: Inhibition of return. Journal of Experimental Psychology: Human Perception \& Performance, 26, 1591-1605. doi:10.1037/0096-1523.26.5.1591

OgaWA, H., TAKeda, Y., \& YAGI, A. (2002). Inhibitory tagging on randomly moving objects. Psychological Science, 13, 125-129. doi:10 $.1111 / 1467-9280.00423$

Pachella, R. G. (1974). The interpretation of reaction time in information-processing research. In B. Kantowitz (Ed.), Human information processing: Tutorials in performance and cognition (pp. 4182). Hillsdale, NJ: Erlbaum.

Posner, M. I., \& Cohen, Y. (1984). Components of visual orienting. In H. Bouma \& D. G. Bouwhuis (Eds.), Attention and performance X: Control of language processes (pp. 551-556). Hove, U.K.: Erlbaum.

Ratcliff, R. (1978). A theory of memory retrieval. Psychological Review, 85, 59-108. doi:10.1037/0033-295X.85.2.59

Shore, D., \& Klein, R. M. (2000). On the manifestations of memory in visual search. Spatial Vision, 14, 59-75. doi:10.1163/ 156856801741369

TAKEDA, Y., \& YAGI, A. (2000). Inhibitory tagging in visual search can be found if search stimuli remain visible. Perception \& Psychophysics, 62, 927-934.

TownSEND, J. T. (1974). Issues and models concerning the processing of a finite number of inputs. In B. H. Kantowitz (Ed.), Human information processing: Tutorials in performance and cognition (pp. 133168). Hillsdale, NJ: Erlbaum

TownsEnd, J. T., \& Ashby, F. G. (1978). Methods of modeling capacity in simple processing systems. In N. J. Castellan, Jr., \& F. Restle (Eds.), Cognitive theory (Vol. 3, pp. 199-239). Hillsdale, NJ: Erlbaum.
Treisman, A. M., \& Gelade, G. (1980). A feature-integration theory of attention. Cognitive Psychology, 12, 97-136. doi:10.1016/0010 $-0285(80) 90005-5$

von Mühlenen, A., Müller, H. J., \& Müller, D. (2003). Sit-andwait strategies in dynamic visual search. Psychological Science, 14, 309-314. doi:10.1111/1467-9280.14441

WANG, Z., \& KLEIN, R. M. (in press). Searching for inhibition of return in visual search: A review. Vision Research.

WiCKELGREN, W. A. (1977). Speed-accuracy trade off and information processing dynamics. Acta Psychologica, 41, 67-85. doi:10.1016/0001 -6918(77)90012-9

Wolfe, J. M. (1994). Guided Search 2.0: A revised model of visualsearch. Psychonomic Bulletin \& Review, 1, 202-238.

Wolfe, J. M., CAVE, K. R., \& Franzel, S. L. (1989). Guided search: An alternative to the feature integration model for visual search. Journal of Experimental Psychology: Human Perception \& Performance, 15, 419-433. doi:10.1037/0096-1523.15.3.419

Wolfe, J. M., \& PoKorny, C. W. (1990). Inhibitory tagging in visual search: A failure to replicate. Perception \& Psychophysics, 48, 357362 .

\section{NOTES}

1. Two of 22 participants in Experiment 1, 1 of 24 participants in Experiment 2, and 1 of 25 participants in Experiment 3 were excluded from analysis because they did not follow the instructions.

2. In Experiments 2 and 3 (dynamic search tasks), targets appeared only at locations (one third of the set size) that were selected before each trial began, and probes were never presented at these locations. This procedure guaranteed that probes were never presented at locations where there had been a target in any preceding frame of a dynamic search trial.

3. Target-absent and target-present trials were subjected to separate ANOVAs, revealing that in neither condition was the interaction between task and probe significant $[F(1,22)=0.11, p=.74 ; F(1,22)=1.96$, $p=.18]$

4. The search performance difference between the static, slow dynamic, and fast dynamic searches was statistically significant. When, instead, we performed these analyses using inverse efficiency (RT/ accuracy), as suggested by Townsend and Ashby (1978), the results were essentially the same.

\section{APPENDIX}

Mean Probe Response Time (RT), Miss, and False Alarm Under Each Condition of Experiments 1-3

\begin{tabular}{|c|c|c|c|c|c|c|c|c|c|c|c|c|c|c|c|c|}
\hline & \multicolumn{8}{|c|}{ Easy Search } & \multicolumn{8}{|c|}{ Difficult Search } \\
\hline & \multicolumn{4}{|c|}{ Target Absent } & \multicolumn{4}{|c|}{ Target Present } & \multicolumn{4}{|c|}{ Target Absent } & \multicolumn{4}{|c|}{ Target Present } \\
\hline & \multicolumn{2}{|c|}{ Set Size 6} & \multicolumn{2}{|c|}{ Set Size 12} & \multicolumn{2}{|c|}{ Set Size 6} & \multicolumn{2}{|c|}{ Set Size 12} & \multicolumn{2}{|c|}{ Set Size 6} & \multicolumn{2}{|c|}{ Set Size 12} & \multicolumn{2}{|c|}{ Set Size 6} & \multicolumn{2}{|c|}{ Set Size 12} \\
\hline & Off & On & Off & On & Off & On & Off & On & Off & On & Off & On & Off & On & Off & On \\
\hline \multicolumn{17}{|l|}{ Experiment 1: Static } \\
\hline Probe RT (msec) & 397 & 415 & 396 & 393 & 370 & 373 & 362 & 374 & 424 & 452 & 414 & 441 & 373 & 389 & 371 & 389 \\
\hline Miss (\%) & 0.83 & 2.08 & 2.92 & 0.83 & 0.83 & 1.67 & 2.92 & 1.25 & 0.42 & 1.25 & 3.33 & 2.92 & 1.25 & 3.75 & 0.83 & 3.33 \\
\hline False alarm (\%) & \multicolumn{2}{|c|}{0.21} & \multicolumn{2}{|c|}{0.42} & \multicolumn{2}{|c|}{1.88} & \multicolumn{2}{|c|}{0.83} & \multicolumn{2}{|c|}{0.83} & \multicolumn{2}{|c|}{0.63} & \multicolumn{2}{|c|}{1.88} & \multicolumn{2}{|c|}{1.25} \\
\hline \multicolumn{17}{|c|}{ Experiment 2: Fast Dynamic } \\
\hline Probe RT (msec) & 413 & 419 & 409 & 404 & 400 & 399 & 401 & 408 & 446 & 457 & 435 & 430 & 404 & 437 & 413 & 423 \\
\hline Miss (\%) & 0.72 & 1.09 & 1.81 & 0.72 & 0.72 & 0.72 & 0.72 & 1.09 & 1.09 & 2.90 & 2.17 & 2.90 & 0.72 & 3.99 & 1.81 & 2.54 \\
\hline False alarm (\%) & \multicolumn{2}{|c|}{0.54} & \multicolumn{2}{|c|}{0.91} & \multicolumn{2}{|c|}{2.90} & \multicolumn{2}{|c|}{4.17} & \multicolumn{2}{|c|}{0.54} & \multicolumn{2}{|c|}{0.54} & \multicolumn{2}{|c|}{1.45} & \multicolumn{2}{|c|}{3.44} \\
\hline \multicolumn{17}{|c|}{ Experiment 3: Slow Dynamic } \\
\hline Probe RT (msec) & 415 & 415 & 392 & 407 & 398 & 398 & 381 & 409 & 414 & 434 & 408 & 432 & 389 & 421 & 389 & 409 \\
\hline Miss (\%) & 0.69 & 0.35 & 1.39 & 0.69 & 0.69 & 2.08 & 0.35 & 1.39 & 0.00 & 0.69 & 0.00 & 0.00 & 1.04 & 2.08 & 0.35 & 2.78 \\
\hline False alarm $(\%)$ & & & & 52 & & & & 74 & & & 1. & 22 & & 39 & & 39 \\
\hline
\end{tabular}

